
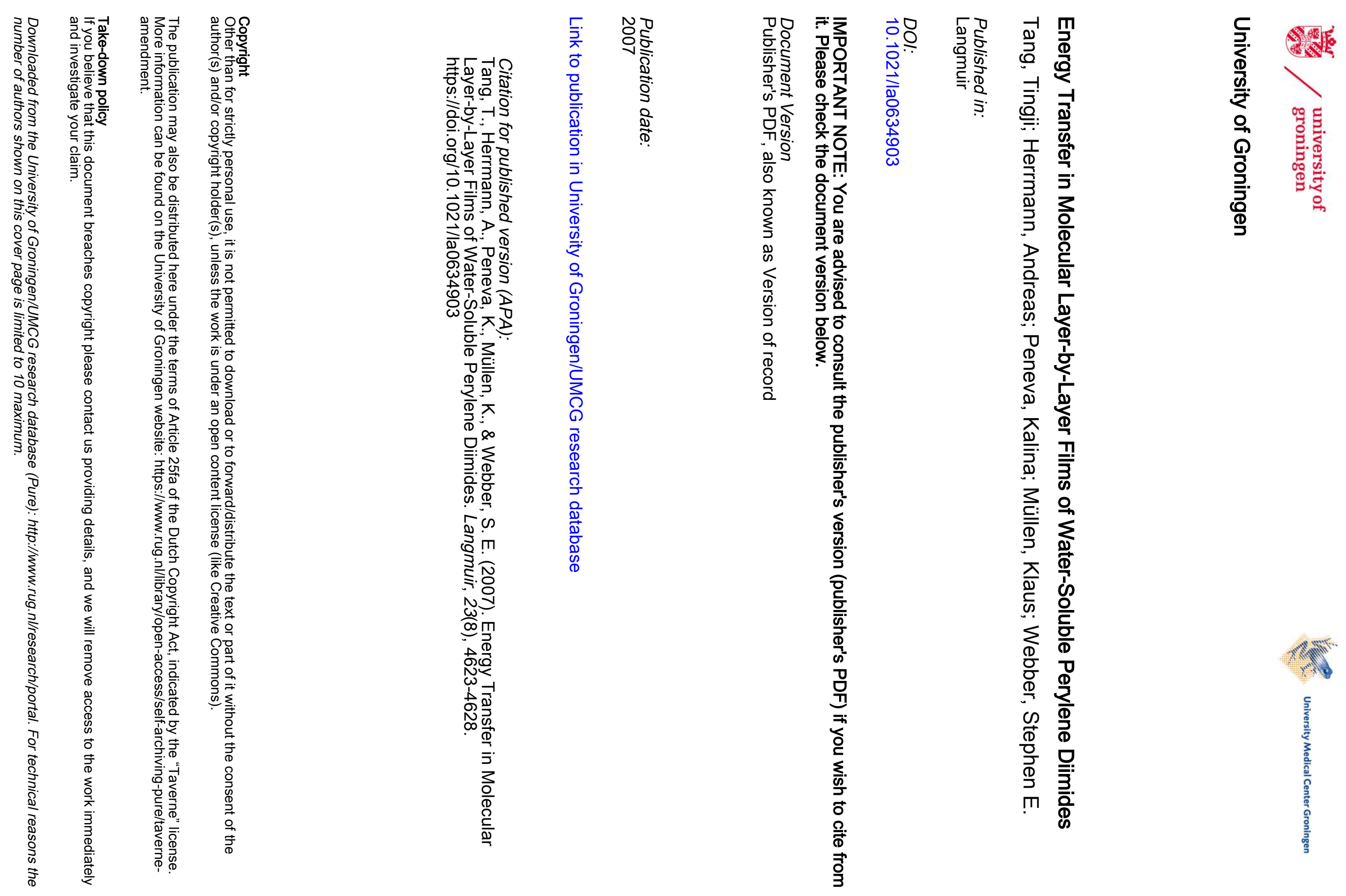


\title{
Energy Transfer in Molecular Layer-by-Layer Films of Water-Soluble Perylene Diimides
}

\author{
Tingji Tang, ${ }^{\dagger}$ Andreas Herrmann, ${ }^{\ddagger}$ Kalina Peneva, ${ }^{\ddagger}$ Klaus Müllen,$\stackrel{\ddagger}{\ddagger}$ and \\ Stephen E. Webber*, ${ }^{*}$ \\ Department of Chemistry and Biochemistry and Center for Nano and Molecular Science, The University of \\ Texas at Austin, Austin, Texas 78712, and Max-Planck-Institut für Polymerforschung, Ackermannweg 10, \\ D-55128 Mainz, Germany
}

Received December 1, 2006. In Final Form: January 29, 2007

\begin{abstract}
Multilayer films of water-soluble anionic and cationic perylene diimide (PDI) moieties have been prepared using the molecular layer-by-layer method described in an earlier publication (Tang, T. J.; Qu, J. Q.; Müllen, K.; Webber, S. E. Langmuir 2006, 22, 26-28) and the fluorescence intensity compared with and without a base layer prepared using an anionic terrylene diimide dye (n-TDI), which serves as an energy-trapping layer for the PDI exciton. The fluorescence quenching data could be fit equally well to a modification of a model used by Kuhn to describe energy transfer from a J aggregate or a model developed by Kenkre and Wong to describe excitonic transfer. For both models, we obtain a characteristic energy-transfer distance on the order of $5.4 \mathrm{~nm}$. Fluorescence quenching of the PDI via a single Förster energy-transfer step to the n-TDI layer is ruled out on the basis of the observed power-law dependence. We also consider a model in which the excitation is trapped at the outermost surface. This model provides a reasonable fit to the data only if the Kuhn relationship is used.
\end{abstract}

\section{Introduction}

Energy transfer within layers of organic chromophores has been studied for more than 50 years and has been motivated by interests as disparate as scintillation counting ${ }^{1}$ to solar-energy conversion. ${ }^{2}$ Much of the earliest work focused on organic crystals, but it has been realized over the past few years that the incorporation of chromophores into layered polyelectrolyte films can also be used to collect and direct photons within the film. ${ }^{3}$ A variety of workers have incorporated chromophores into the so-called "layer-by-layer" (LBL) method, in which alternate layers of polyelectrolytes of opposite charge are deposited on a substrate by simple dipping into an aqueous solution. ${ }^{4}$ This method is simple to apply, and the incorporation of charged chromophores is easy to accomplish although quite often a small charged molecule is stripped from the film by subsequent depositions.

We have recently discussed a modification of the LBL method, described as a "molecular layer-by-layer" (MLBL) approach, in which anionic and cationic perylene diimide dyes are deposited sequentially onto a surface without subsequent loss in the next dipping cycle. ${ }^{5}$ We believe that a contributing factor to the stability of these films is the strong $\pi-\pi$ stacking interaction between the perylene rings, which unfortunately also serves to reduce the fluorescence quantum yield substantially. A similar effect has been noted by Dobrawa et al. for coordination polyelectrolytes containing perylene diimides in the backbone of the chain. ${ }^{6}$ Perylene diimides have been heavily studied because of their strong absorption in a convenient spectral range, generally high fluorescence quantum yields (at least in solution), and photo-

\footnotetext{
$\dagger$ The University of Texas at Austin.

$\$$ Max-Planck-Institut für Polymerforschung

(1) Birks, J. B. Scintillation Counters; McGraw-Hill: New York, 1953.

(2) Gregg, B. A. J. Phys. Chem. B 2003, 107, 4688.

(3) (a) Kaschak, D. M.; Lean, J. T.; Waraksa, C. C.; Saupe, G. B.; Usami, H.; Mallouk, T. E. J. Am. Chem. Soc. 1999, 121, 3435. (b) Dai, Z.; Dähne, L.; Donath, E.; Möhwald, H. J. Phys. Chem. B 2002, 106, 11501.

(4) (a) Decher, G., Hong, J. Makromol. Chem. Symp. 1991, 46, 321. (b) Decher, G. Science 1997, 277, 1232 .

(5) Tang, T.-J.; Qu, J-Q.; Müllen, K.; Webber, S. E. Langmuir 2006, 22, 26. (6) Dobrawa, R.; Kurth, D. G.; Würthner, F. Polym. Prepr. 2004, 45, 378.
}

stability. Films of perylene diimides, typically vapor deposited, have been used in photosensitization applications (reviewed by $\mathrm{Law}^{7}$ ), but materials based on these compounds often have highly variable spectra because of the perturbations induced by strong $\pi-\pi$ stacking. In some cases, the concentration-induced changes in the solution-phase absorption and fluorescence spectra are spectacular. ${ }^{8,9}$ A recent review by Würthner provides a concise discussion of the aggregations and photophysical properties of bay-substituted PDIs and the general type discussed herein. ${ }^{10}$ For our materials and the MLBL technique, there is no appreciable shift in the absorption or fluorescence spectra upon film formation, although we do believe that there is appreciable $\pi$ stacking based on the fluorescence quenching. In general, the challenge for PDIs is to control the degree of $\pi$ stacking in order achieve good energy- or charge-transfer properties without completely quenching the excited states or creating energy- or charge-trapping states.

The present experiments are designed to examine heterofilms in which a water-soluble terrylene diimide (n-TDI) compound serves the dual purpose as a base layer upon which oppositely charged water-soluble perylene diimides (n-PDI and p-PDI) can be deposited (chemical structures in Scheme 1) and as an energy trap. The efficiency of energy transfer $\left(\Phi_{\mathrm{ET}}\right)$ to the n-TDI was measured as a function of the number of n-PDI/p-PDI bilayers $(n)$ and drops to 0.5 for approximately nine bilayers. In the Results section, we discuss the fitting of the $n$ dependence to several different models: (1) a trapped exciton transferring to a plane of acceptors, (2) a trapped J aggregate (coherently delocalized exciton) transferring to a plane of acceptors, or (3) a randomwalk exciton migration to a strongly quenching surface. These latter two models provide the best fit to our data with a characteristic distance of energy transfer on the order of $6 \mathrm{~nm}$,

(7) Law, K.-Y. Chem. Rev. 1993, 93, 449

(8) Wang, W.; Han, J. J.; Wang, L.-Q.; Li, L.-S.; Shaw, W. J.; Li, A. D. Q. Nano Lett. 2003, 3, 455 .

(9) Würthner, F.; Chen, Z.; Volker Dehm, V.; Stepanenko, V. Chem. Commun. 2006, 1188.

(10) Würthner, F. Pure Appl. Chem. 2006, 78, 2341. 

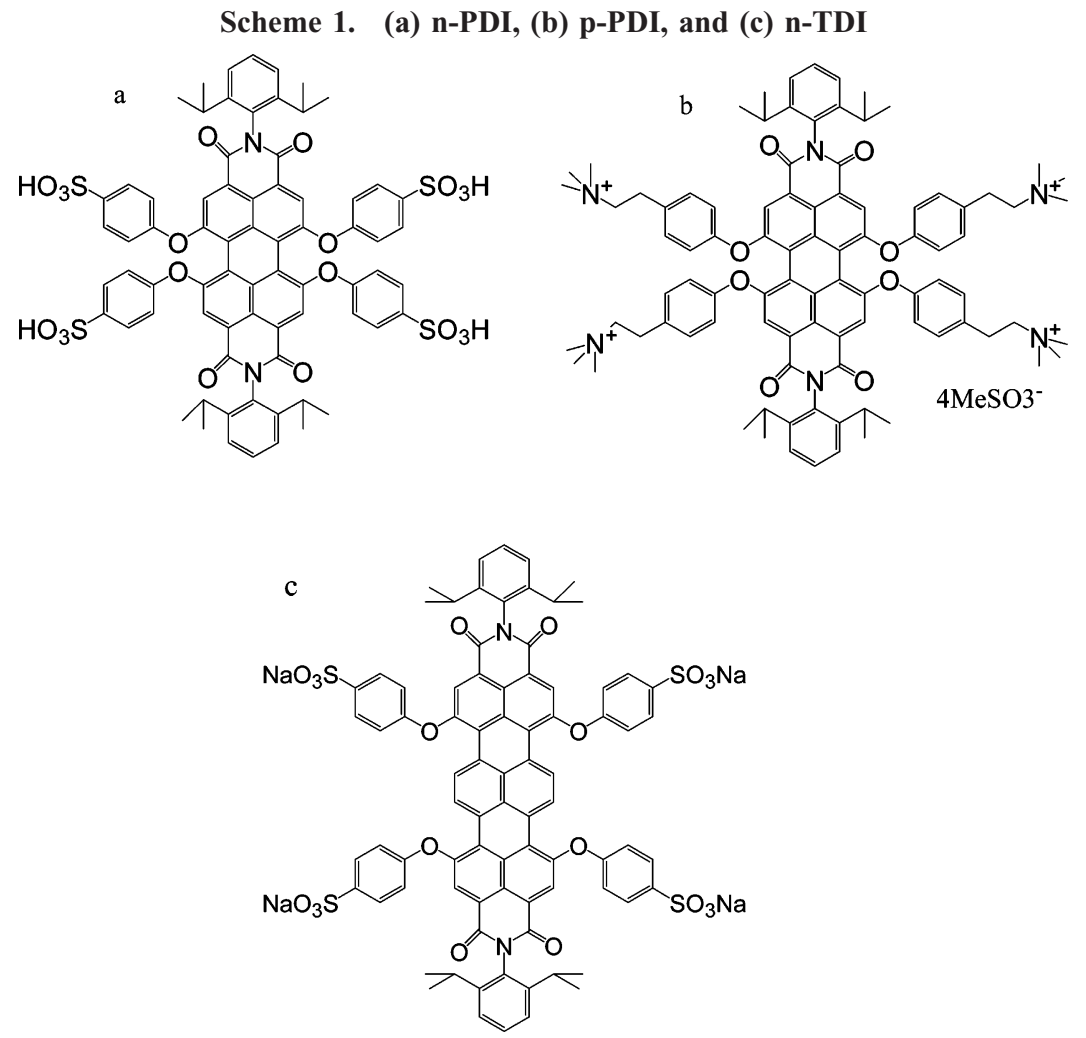

which is much smaller than has been reported for annealed films of other perylene diimide derivatives. ${ }^{11,12}$

\section{Experimental Section}

Materials. The chemical structures of p-PDI (CAS [81720711-7]), n-PDI (CAS [694438-88-5]), and n-TDI (which is the sodium salt of the parent tetrasulfonic acid, CAS [862852-56-0]) are shown in Scheme 1. These compounds were synthesized by Müllen et al. ${ }^{13,14}$ ( $\mathrm{p}$ - and n-PDI are the same materials used in our earlier report concerning the MLBL method..$^{5}$ ) The PDI peak molar extinction coefficients in water were measured to be $3.28 \times 10^{4}$ and $2.97 \times$ $10^{4} \mathrm{M}^{-1} \mathrm{~cm}^{-1}$ for $\mathrm{p}$-PDI $(\lambda=591 \mathrm{~nm})$ and $\mathrm{n}$-PDI $(\lambda=568 \mathrm{~nm})$, respectively. Both of these compounds are fluorescent in the aqueous phase $\left(\phi_{\mathrm{fl}}^{\mathrm{n}-\mathrm{PDI}}=0.54\right.$ and $\left.\phi_{\mathrm{fl}}{ }^{\mathrm{p}-\mathrm{PDI}}=0.14\right)$. However, if $\mathrm{n}-\mathrm{PDI}$ and p-PDI are mixed in a 1:1 ratio, then the fluorescence is strongly quenched (by a factor of ca. 13 relative to $\mathrm{p}$-PDI at an equivalent optical density), presumably because of the formation of aggregates. Analogous quenching also occurs in the MLBL assembly. n-TDI has an extinction coefficient of $2.9 \times 10^{4} \mathrm{M}^{-1} \mathrm{~cm}^{-1}(\lambda=640 \mathrm{~nm})$, and we find it to be fluorescent in water only in the presence of surfactants (with dodecyl trimethyl ammonium chloride $\phi_{\mathrm{fl}}{ }^{\mathrm{n}-\mathrm{TDI}} \approx$ 0.03). Jung et al. have made similar observations for this compound. ${ }^{15}$

The fluorimeter used was a SPEX Fluorolog- $\tau 2$ equipped with a $450 \mathrm{~W}$ xenon light source, Czerny-Turner double grating excitation, and emission monochromators. A photomultiplier voltage of $950 \mathrm{~V}$ was typically used, and the excitation and emission slit widths were set at $2 / 2 / 4 / 4 \mathrm{~mm}$ for film samples. The fluorescence emission spectra were recorded in the front-face mode under ambient condition (e.g., no purge with inert gas was found to be necessary). All of the fluorescence spectra were recorded with excitation at $540 \mathrm{~nm}$.

(11) Gregg, B. A.; Sprague, J.; Peterson, M. W. J. Phys. Chem. B 1997, 101 , 5362.

(12) Adams, D. M.; Kerimo, J.; O'Connor, D. B.; Barbara, P. F. J. Phys. Chem. A 1999, 103, 10138 .

(13) (a) Qu. J.; Zhang, J.; Drimsdale, A. C.; Müllen, K.; Jaiser, F.; Yang, X.; Neher, D. Macromolecules 2004, 37, 8197. (b) Kohl, C.; Weil, T.; Qu, J.; Müllen, K. Eur. J. Chem. 2004, 10, 5297.

(14) Qu, J.; Kohl, C.; Pottek, M.; Müllen, K. Angew. Chem. 2004, 116, 1554.

(15) Jung, C.; Müller, B. K.; Lamb, D. C.; Nolde, F.; Müllen, K.; Bräuchle, C. J. Am. Chem. Soc. 2006, 128, 5283.
Table 1. FRET Parameters

\begin{tabular}{cccc}
\hline donor-acceptor & $\begin{array}{c}J\left(\times 10^{-14}\right) \\
\left(\mathrm{cm}^{3} \mathrm{M}^{-1}\right)^{\mathrm{a}}\end{array}$ & $Q_{\mathrm{D}}{ }^{b}$ & $\begin{array}{c}R_{0}{ }^{c} \\
(\mathrm{~nm})\end{array}$ \\
\hline p-PDI/p-PDI & 7.99 & 0.14 & 3.58 \\
n-PDI/n-PDI & 8.37 & 0.53 & 4.50 \\
p-PDI/n-PDI & 5.78 & 0.14 & 3.39 \\
n-PDI/p-PDI & 8.37 & 0.53 & 4.86 \\
p-PDI/n-TDI & 27.9 & 0.14 & 4.46 \\
n-PDI/n-TDI & 27.9 & 0.53 & 7.46 \\
${ }^{a} J=\int_{0}^{\infty} \frac{F_{\mathrm{D}}(v) \epsilon_{\mathrm{A}}(v)}{v^{4}} \mathrm{~d} v$. & & \\
& & & \\
& &
\end{tabular}

escence. ${ }^{c} R_{0}{ }^{6}=\frac{9000(\ln 10) \kappa^{2} Q_{\mathrm{D}}}{128 \pi^{4} N n^{4}} J$ and taking $\kappa^{2}=\frac{2}{3}, n=1.57$.

Förster $\boldsymbol{R}_{\mathbf{0}}$ Values. The n- and p-PDI compounds have sufficiently large transition dipole moments and spectral overlaps of their absorption and fluorescence spectra that the Förster $R_{0}$ value for energy transfer lies in the range of 3.4-4.9 nm (assuming $\kappa^{2}$, the average orientational factor, has a value of $2 / 3$, which is appropriate for the solution phase; for static and randomly oriented molecules, the appropriate value is $\left.0.476^{16}\right)$. The estimated $R_{0}$ value for $\mathrm{n}$ - or p-PDI to $\mathrm{n}$-TDI is even larger at $4.5-7.5 \mathrm{~nm}$ (Table 1). As can be seen in Figure 1, there is excellent spectral overlap of the p-PDI fluorescence with the n-TDI absorption.

Molecular Layer-by-Layer Procedures. The quartz substrates were dipped in fresh piranha solution for $10 \mathrm{~min}\left(7: 3 \mathrm{v} / \mathrm{v} \mathrm{H}_{2} \mathrm{SO}_{4} /\right.$ $\left.\mathrm{H}_{2} \mathrm{O}_{2}\right)$ followed by a RCA solution for $10 \mathrm{~min}\left(6: 2: 2 \mathrm{v} / \mathrm{v} / \mathrm{v} \mathrm{NH} 4^{-}\right.$ $\mathrm{OH} / \mathrm{H}_{2} \mathrm{O}_{2} / \mathrm{H}_{2} \mathrm{O}$ ), both at $60{ }^{\circ} \mathrm{C}$, and then baked at $420{ }^{\circ} \mathrm{C}$ for $6 \mathrm{~h}$. (Caution! Piranha solution is extremely corrosive.) The advancing contact angle with water was measured to be approximately $5^{\circ}$ for all of the substrates.

Energy-acceptor molecule n-TDI was absorbed onto the bare cleaned quartz substrate as the foundation layer $(0.1 \mathrm{~g} / \mathrm{L}, 12 \mathrm{~min}$ of dipping time, three washes with DI water). Films of (p-PDI/n-

(16) (a) Birks, J. B. Photophysics of Aromatic Molecules; Wiley-Interscience: New York, 1970. (b) Lakowicz, J. R. Principles of Fluorescence Spectroscopy, 2nd ed.; Kluwer Academic/Plenum Publishers: New York, 1999. 


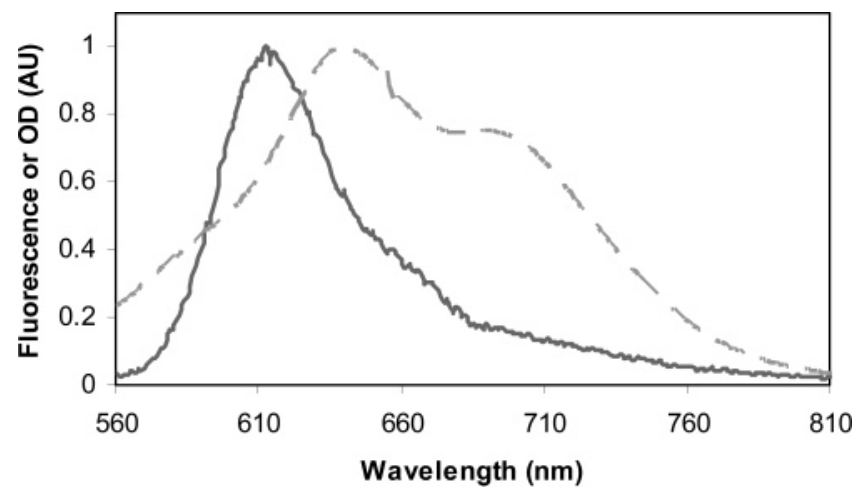

Figure 1. Illustration of the overlap between the p-PDI fluorescence $(-)$ and n-TDI absorption (--) in arbitrary units.
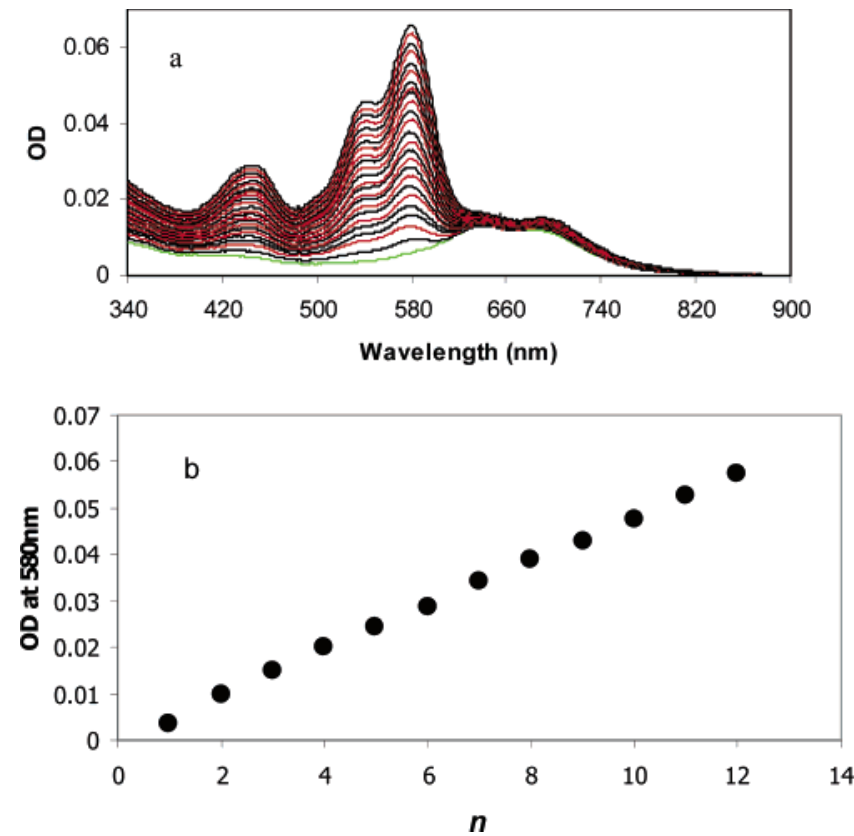

Figure 2. (a) Buildup of the absorption spectrum of p-PDI and n-PDI monolayers on a base of n-TDI. (b) Peak absorbance at 580 $\mathrm{nm}$ as a function of monolayers p-PDI (see text) after correcting for the absorbance of n-TDI (green, TDI only; black, added p-PDI; red, added n-PDI).

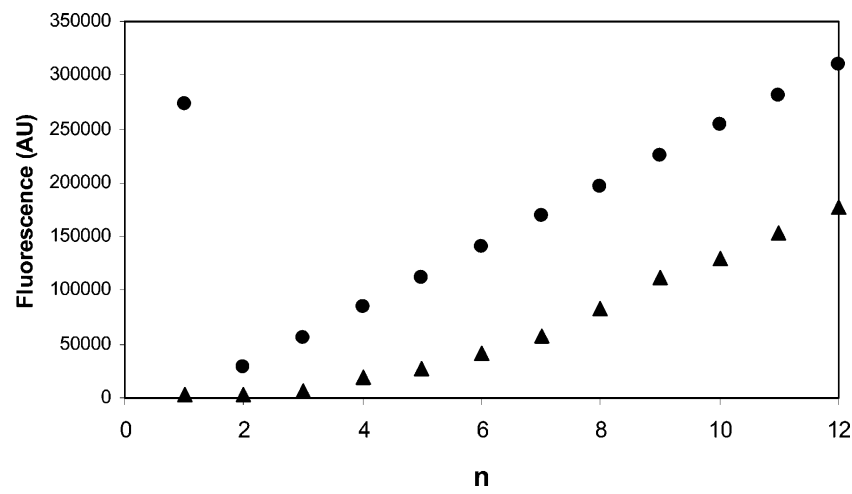

Figure 3. Comparing the fluorescence of p-PDI(n-PDI/p-PDI $)_{n-1}$ $(\bullet)$ and $\mathrm{n}-\mathrm{TDI} / \mathrm{p}-\mathrm{PDI}(\mathrm{n}-\mathrm{PDI} / \mathrm{p}-\mathrm{PDI})_{n-1}(\mathbf{\Delta})$. Note that $n$ corresponds to the number of p-PDI layers in the film $\left(\lambda_{\mathrm{ex}}=540 \mathrm{~nm}\right)$.

PDI $)_{n-1} /$ p-PDI (for simplicity p-PDI was always the outside layer in the plots of intensity vs the number of layers in Figures 2 and 3) were then built up on the n-TDI foundation layer by dipping the substrates alternately into a $0.1 \mathrm{~g} / \mathrm{L}$ p-PDI or n-PDI solution for 10 min, starting with the p-PDI layer. After each dipping, there were three washes with DI water to remove the physically absorbed PDI dye. After this step, a gentle stream of argon gas was used to dry

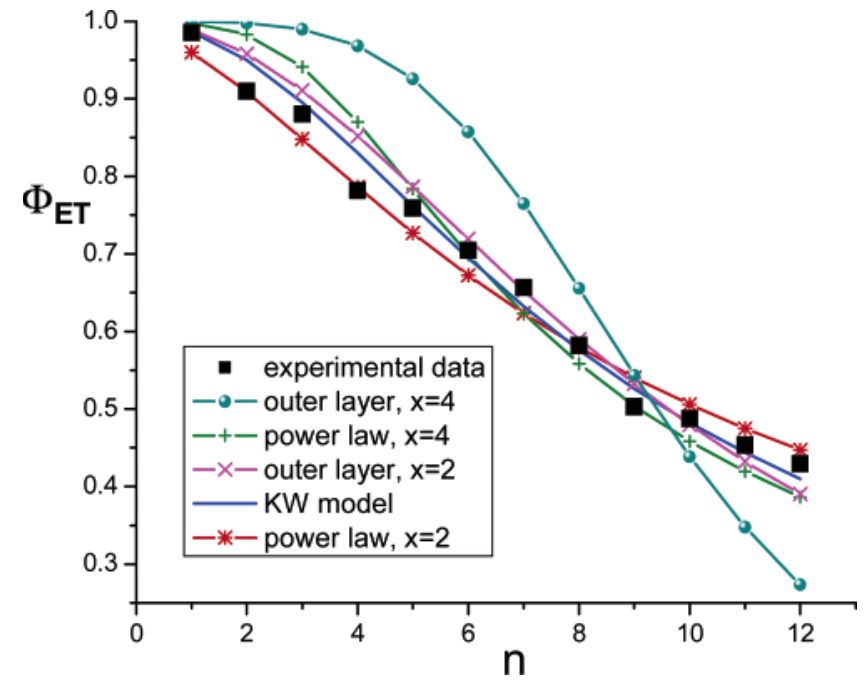

Figure 4. Experimental values of $\Phi_{\mathrm{ET}}$ compared with the best fit of different models discussed in the text.

the films before they were put into the next dye solution. These routines were repeated until the desired number of double layers was achieved. The spectra after each cycle (Figure 2a) demonstrated a continuous buildup of PDI absorption and no loss of n-TDI absorption. The absorbance at $580 \mathrm{~nm}$ (correcting for the contribution from n-TDI) was linear in the number of deposition cycles (Figure $2 b)$. The PDI loading for each layer deposition is about the same as that for (p-PDI/n-PDI) ${ }_{n}$ discussed previously. ${ }^{5}$ In this previous publication, we noted an alternation of fluorescence intensity according to which PDI species was the outermost layer, and the same is true for these films. Our fluorescence quenching by TDI is the same regardless of the outer layer, and the results presented herein are for p-PDI as the outer layer only.

We assume that our MLBL films are composed of discrete noninterpenetrating layers of PDI monolayers, which were determined by ellipsometry to be about $0.6 \mathrm{~nm}$ thick $\left(d_{\mathrm{PDI}}\right)$. Therefore, PDI film thickness $d$ for the $n$th p-PDI layer (in nanometers) can be estimated as follows:

$$
d_{n}=[2(n-1)-1] d_{\mathrm{PDI}}=1.2 n-0.6
$$

\section{Results: Energy-Transfer Efficiency for n-TDI/(p-PDI/ $\mathrm{n}-\mathrm{PDI})_{n}$ and Fit to Models}

In this study, we wished to determine if the energy transfer from the PDI-multilayer to the n-TDI monolayer was efficient and if the dependence of the energy-transfer efficiency on the number of layers could be fit to any simple model. Experimentally, the energy-transfer efficiency was obtained by comparing the total PDI fluorescence of an n-TDI/(p-PDI/n-PDI $)_{n-1}(\mathrm{p}-\mathrm{PDI})$ multilayer $\left(I_{\mathrm{TDI}}\right)$ with that of a $(\mathrm{p}-\mathrm{PDI} / \mathrm{n}-\mathrm{PDI})_{n-1}(\mathrm{p}-\mathrm{PDI})$ multilayer $\left(I_{0}\right)$ (Figure 3 ). The absorption of n-TDI does not interfere with the excitation of n-PDI or p-PDI, and the optical density of the films is very low, such that the film can be considered to be uniformly excited and there is no appreciable absorption of the PDI fluorescence by $n-T D I(O D \leq 0.01)$. The energy-transfer efficiency is therefore estimated by

$$
\Phi_{\mathrm{ET}}=1-\frac{I_{\mathrm{TDI}}}{I_{0}}
$$

$\Phi_{\mathrm{ET}}$ as a function of $n$ is plotted in Figure 4. As can be seen, the energy transfer drops from essentially unity for $n=1$ to ca. 0.45 for $n=12$ 
Table 2. Best-Fit Parameters to $\Phi_{\mathrm{ET}}^{\text {expt }}$ (Smallest Values Underlined)

\begin{tabular}{|c|c|c|c|c|}
\hline model & $n_{0}$ & std error $^{c}$ & $\begin{array}{c}\text { sum of weighted } \\
\text { deviations }^{d}\end{array}$ & slope $^{e}$ \\
\hline $\begin{array}{l}\text { outer layer, } x=4 \\
\text { (see eq } 3 \text { ) }\end{array}$ & $9.4^{a}$ & 0.118 & -0.583 & +0.0343 \\
\hline $\begin{array}{l}\text { outer layer, } x=2 \\
\text { (see eq } 3 \text { ) }\end{array}$ & $9.6^{a}$ & 0.032 & -0.148 & +0.0089 \\
\hline $\begin{array}{l}\text { power law, } x=4 \\
\text { (see eq } 4 \text { ) }\end{array}$ & $4.7^{b}$ & 0.044 & +0.0298 & +0.0152 \\
\hline $\begin{array}{l}\text { power law, } x=2 \\
\text { (see eq 4) }\end{array}$ & $4.9^{b}$ & 0.025 & +0.0032 & -0.0075 \\
\hline KW model & $5.0^{b}$ & 0.022 & +0.0297 & $\underline{-0.0051}$ \\
\hline
\end{tabular}

${ }^{a}$ Fitting to the number of layers (estimated error \pm 0.2 ). ${ }^{b}$ Fitting to the number of layers (estimated error \pm 0.1 ). ${ }^{c}$ Square root of the sum of the deviations squared divided by $12 .{ }^{d}$ Sum of $\left(\Phi_{\mathrm{ET}}^{\text {expt }}-\Phi_{\mathrm{ET}}^{\text {calc }}\right) / \Phi_{\mathrm{ET}}^{\text {expt }}$ for 12 data points. ${ }^{e}$ Fit of weighted deviations to a straight line. The smallest slope means the least correlation (Supporting Information, Figure S1).

The efficiency of energy transfer from a layer of donors to a layer of acceptors with separation distance $d$ is typically expressed by the equation

$$
\Phi_{\mathrm{ET}}=1-\left(1+\left(\frac{d_{0}}{d}\right)^{x}\right)^{-1}
$$

In this expression, $d_{0}$ is the characteristic distance between donor and acceptors corresponding to a $50 \%$ probability of energy transfer, and $x$ depends on the geometry and model assumed. For example, $x$ is equal to 4 in the case of a plane of donor molecules transferring energy to a plane of acceptor molecules, ${ }^{17}$ and $x$ is equal to 2 in the case of energy transfer from a J-type aggregate to acceptors in a planar array. ${ }^{18,19} \mathrm{In}$ this latter case, the excitation is strongly coupled between some number of individual chromophores, and the length of this aggregate is significant compared to $d$.

In our earlier paper, we observed an alternation of fluorescence intensity that depended on which particular PDI compound comprised the outer layer. We postulated that this was a consequence of energy transfer to the outer layer which, for unknown reasons, was acting as an exciton trap. In that case, eq 3 can be used to describe $\Phi_{\mathrm{ET}}$, with $x=4$ for the case in which the excitation is localized and $x=2$ if the excitation is coherently delocalized (like a J aggregate) (referred to as "outer layer $x=$ 2" (or 4) in Figure 4). The best fit that could be achieved for $x$ $=4$ was very poor, and we dismiss this model from further consideration (blue curve with symbols in Figure 4; see Table 2 for the fitting parameters and standard deviations for all models considered). The fit is reasonably good for $x=2$ (Figure 4 , magenta curve, and Table 2), although on the basis of the standard deviation, it is not as good as for the models described next. In Table 2, we also present the sum of weighted residuals as a measure of the randomness of the deviation of the model from the experimental data and the least-squares fit of the slope of the weighted deviations as a function of $n$ (plot of the weighted deviations in Supporting Information). The smaller the magnitude of this slope, the smaller the correlation of the weighted deviations.

In our system, we have $n$ bilayers of PDI donors that are homogeneously excited, so if we neglect interlayer energy transfer, we can take $\Phi_{\mathrm{ET}}$ to be an average over $n$

(17) Lowman, G. M.; Daoud, N.; Case, R. M.; Carson, P. J.; Buratto, S. K Nano Lett. 2001, 1, 667.

(18) (a) Kuhn, H. J. Chem. Phys. 1970, 53, 101. (b) Nakahara, H.; Fukuda K.; Möbius, D.; Kuhn, H. J. Phys. Chem. 1986, 90, 6144

(19) Saha, D. C.; Bhattacharjee, D.; Misra, T. N. Opt. Mater. 1998, 10, 285

$$
\Phi_{\mathrm{ET}}=1-\frac{1}{n_{i=1}} \sum_{1+\left(d_{0} / i d\right)^{x}}
$$

where $d$ is the thickness of a bilayer (approximately $1.2 \mathrm{~nm}$ according to eq 1). The best fit to our experimental data with $x$ $=2$ and 4 is given in Figure 4 (red and green curves with symbols, respectively, referred to as "power law $x=2$ " (or 4)), and the parameters and standard deviations are given in Table 2. The best fit for $x=4$ systematically deviates from the experimental data whereas $x=2$ provides a more satisfactory fit.

Kenkre and Wong (KW) have discussed exciton migration studies with "end detectors" using a random-walk master equation approach in which it is assumed that excitons are created within a slab for which one side contains a "detector layer" (a substance that can quench the mobile exciton) and the other side is a perfect reflector for the exciton. ${ }^{20}$ Their treatment can be applied to optically thick materials and has been used extensively in an earlier study of excitonic transport in perylene diimide layers. ${ }^{11}$ One of the main points of the KW model is to consider the effect on exciton quenching when the rate of quenching at the detector layer (n-TDI in our case) is not very fast. They present the following equation for the quantum efficiency of exciton quenching in the case of an optically thin sample:

$$
\Phi_{\mathrm{ET}}^{\mathrm{KW}}=\Phi_{\mathrm{ET}}^{*} \frac{\mathcal{T} / F}{(\mathcal{T} / F)+c}
$$

where

$$
c=\frac{1+f \tau_{\text {trap }}}{\sqrt{F \tau_{\mathrm{ex}}}} \frac{\sinh (N \xi)}{\cosh [(N-(1 / 2)) \xi\}}
$$

$\mathcal{T} / F$ is the ratio between the rate of transfer of the exciton to the trap $(\mathcal{T})$ and the intersite exciton transfer rate $(F), f$ is the rate of escape of the exciton from the trap, and $\tau_{\text {ex }}$ and $\tau_{\text {trap }}$ are the excited-state lifetimes for the exciton and trap excited state, respectively. In eq $5, \Phi_{\mathrm{ET}}^{*}$ is the quantum yield of energy transfer in the case in which the trapping rate is much faster than the exciton transfer rate (i.e., $\mathcal{T} / F \rightarrow \infty$ ). If $F \tau_{\text {ex }}$ is large (i.e., the square of the exciton mean free path is much larger than 1 ), then

$$
N \xi=\frac{1}{\sqrt{F \tau_{\text {ex }}}}
$$

and

$$
\Phi_{\mathrm{ET}}^{*}=\frac{d_{0}}{D} \tanh \left(\frac{D}{d_{0}}\right)
$$

In this equation, $d_{0}$ is the exciton mean-free path $\left(\sqrt{F \tau_{\mathrm{ex}}} a\right)$, and $D$ is the thickness of the slab $(N a)$, where $a$ is the lattice spacing. We assume that we can use this "perfect quencher" limit and find that we can fit our data very well to $\Phi_{\mathrm{ET}}^{*}$ (Figure 4 , solid blue curve, referred to as the KW model, and Table 2).

\section{Discussion}

Before we discuss our fluorescence quenching results, we review some of the previously observed properties of n-PDI/ p-PDI multilayers in the absence of n-TDI. ${ }^{5}$ First, the absorption of these films increases linearly with the number of dipping cycles and is essentially identical to a simple sum of the solutionphase spectra, in contrast to many PDI derivatives for which the

(20) Kenkre, V. M.; Wong, Y. M. Phys. Rev. B 1980, 22, 5716. 
absorption spectra in films are often structureless and strongly red-shifted from the spectra of the dilute-solution phase. ${ }^{7,11}$ Even for relatively soluble PDI derivatives, the absorption spectra can exhibit concentration-dependent spectral shifts. ${ }^{8,9}$ Such strong effects are not observed for our PDI compounds in water, but there is evidence for some degree of aggregation in water. ${ }^{21,22}$ MD simulations suggest that roughly cofacial (n-PDI) $)_{2}$ or ( $p-$ $\mathrm{PDI})_{2}$ dimers can form in water but the angle formed between the PDI planes and their center-to-center vectors is inappropriate for strong excited-state dipolar coupling. ${ }^{21}$ Thus, it is possible that we are depositing a mixture of monomeric and dimeric moieties (higher-order aggregates cannot be ruled out although no aggregates could be observed by dynamic light scattering, in contrast to the results reported by Wang et al. ${ }^{8}$ ). Chen et al. have made a very extensive study of bay-substituted PDI in organic solvents and have demonstrated that their aggregation behavior and photophysics are very sensitive to the structural and chemical properties of the bay substituents. ${ }^{23}$

The fluorescence spectra of the n-PDI/p-PDI multilayers are also essentially unchanged from a simple sum of the components (which are very similar to each other) and scale linearly with the number of dipping cycles except for the first layer, which has a much higher quantum yield than the subsequent bilayers. This can be easily understood as the effect of $\pi$ stacking between n-PDI/p-PDI layers, whose formation is encouraged by electrostatic interactions and whose geometry can be expected to be different than that of the PDI homodimers mentioned above. Mixing n-PDI and p-PDI in aqueous solution resulted in a ca. 13-fold decrease in fluorescence intensity, although without a significant change in the fluorescence spectral shape, similar to what we observe in the n-PDI/p-PDI multilayers. ${ }^{5}$ There is also a peculiar alternation of fluorescence intensity according to which PDI species forms the outer layer. (For simplicity, the data in Figure 3 has p-PDI as the outer layer.) We have interpreted this alternation as the result of energy transfer to the outer layer, but the present results cast some doubt on this interpretation, as will be discussed below. It is significant that the fluorescence intensity scales linearly with the number of bilayers and the spectral shape does not change. This suggests that we do not accumulate quenching defects as the number of deposited layers increases.

We have found that we can fit our data most satisfactorily to either the Kuhn model, developed for energy transfer for $\mathrm{J}$ aggregates $^{18}$ and modified by averaging over each layer in the array, or the Kenkre-Wong model of excitonic transfer to a quenching layer. ${ }^{20}$ The best fit for each model yields about the same values of the number of layers $\left(n_{0} \approx 5\right)$ and distance $\left(d_{0}\right.$ $=d n_{0} \approx 6 \mathrm{~nm}$, or more precisely using eq $1,5.4 \mathrm{~nm}$ ) over which energy transfer occurs, but the physical interpretation of these values is quite different.

According to Kuhn et al., ${ }^{18}$

$$
d_{0}=\frac{\alpha}{L}\left(\frac{\lambda_{\mathrm{em}}}{n}\right)^{2}\left(\mathrm{OD}_{\mathrm{A}} q_{\mathrm{D}}\right)^{1 / 2}
$$

where $\alpha$ is a geometric factor whose value is estimated to be $\sim 0.016, \lambda_{\mathrm{em}} / n$ is the wavelength of the emission maximum divided by the refractive index of the medium $(610 \mathrm{~nm} / 1.57$ in our case $)$, $q_{\mathrm{D}}$ is the quantum yield of the donor (in the range of $(0.14-$ $0.5) / 13$ in our case), and $\mathrm{OD}_{\mathrm{A}}$ is the optical density of the acceptor at $\lambda_{\mathrm{em}}$ (ca. 0.015 in our case). $L$ is the length of the aggregate over which the excitation is coherently delocalized, and with $d_{0}$

(21) Tang, T.; Qu, J.; Müllen, K.; Webber, S. E. Langmuir 2006, 22, 7610.

(22) Tang, T. On-going work.

(23) Chen, Z.; Baumeister, U.; Tschierske, C.; Würthner, F. Chem.-Eur. J. 2007, 13, 450 . $\approx 6 \mathrm{~nm}$ and the above parameters we obtain $L$ in the range of $5.1-9.7 \mathrm{~nm}$ (depending on the value of $q_{\mathrm{D}}$ assumed), corresponding to two to four PDI molecules on the basis of their long-axis dimension. Unlike classical J-aggregate spectra, our fluorescence spectra do not display a strong red shift and narrowing of the PDI fluorescence. Saha et al. in their study of energy transfer between 1,2-diphenyl indole (DPI, energy donor) and 9,10-diphenylanthracene (DPA, energy acceptor) confined to different layers of LB films observe an $x=2$ power law (eq 3 ) while observing only a modest red shift and broadening of the DPI fluorescence. ${ }^{19}$ Thus, it seems possible for an excitation to be coherently delocalized without necessarily undergoing a dramatic spectral shift. We note that we obtained a fair fit of the $x=2$ power law to a model in which the excitation is confined to the outer layer but a value of $d_{0}$ that is approximately twice as large (Table 2), which would lead to approximately twice the above-estimated value of $L$. Such a significant delocalization could be the origin of a surface trapping state and would suggest that the PDI moieties self-organize at the outermost layer. However, the fit to this model is slightly inferior to the fit to the power-law model with a homogeneous distribution of excitation (eq 4).

The Kuhn model as we have applied it neglects exciton migration from the point of excitation. The Kenkre-Wong (KW) theory ${ }^{20}$ considers a tight-binding exciton model, and $d_{0}$ in eq 8 corresponds to the exciton diffusion length

$$
d_{0}=\sqrt{F \tau_{\mathrm{ex}}} a
$$

where $a$ is the separation between lattice sites. In our system, we associate $a$ with the bilayer thickness (ca. $1.2 \mathrm{~nm}$ ), but the appropriate "lattice spacing" in the plane of the films is unknown. In the KW model, the quenching process occurs at a rate at which the exciton is one lattice spacing away from the quenching layer. Because n-TDI can quench at larger distances via Förster energy transfer, the KW model is not perfectly appropriate to the present system. Likewise, it is assumed that the exciton is perfectly reflected from the quencher-free surface (i.e., the outermost layer in our system), unlike our earlier proposal that the excitation is trapped at the outer surface. The KW expression that we have used assumes that $\mathcal{T}_{F} \gg 1$, which we believe is reasonable for our experiment, but as Kenkre and Wong point out, the true value of $d_{0}$ may be larger than the value we calculate. If we use our value of $d_{0}$, take $a=1.2 \mathrm{~nm}$, and assume that $\tau_{\mathrm{ex}} \approx 5 \mathrm{~ns} / 13$ ( $5 \mathrm{~ns}$ is the approximate solution-phase lifetime of our PDI compounds, ${ }^{22}$ and the factor of 13 is the approximate fluorescence quenching factor in the MLBL films), then we obtain an estimate for $F$ of $6.5 \times 10^{10} \mathrm{~s}^{-1}$.

Gregg et al. presented an extensive study of exciton migration in vapor-deposited and methylene chloride vapor-annealed films of perylene bis(phenethylimide) (PPEI), which do not have any chemical substituents on the bay regions and exhibit a broadened and red-shifted absorption and fluorescence spectrum in films compared to the spectra for soluble model compounds. ${ }^{11}$ Using the KW model and optically thick samples, these workers estimated a singlet exciton diffusion length of $2.5( \pm 0.5) \mu \mathrm{m}$. Adams et al. also studied similar PPEI films using the NSOM technique and estimated an exciton diffusion length in the range of 50-500 nm. ${ }^{12}$ Evidently, the details of sample preparation can exert quite a large influence on the exciton migration, which is not surprising if disorder plays a significant role in these processes. We assume that our relatively small values of $d_{0}$ are the result of a lack of long-range order and perhaps a nonoptimal average orientation of neighboring PDI moieties. Certainly the work of Gregg et al. ${ }^{11}$ and Adams et al. ${ }^{12}$ demonstrates that 
spectral shifts and broadening do not necessarily lead to quenching of exciton migration. Additionally, the stacking of our baysubstituted PDIs is expected to be quite different than that of PDEI, according to recent studies by Würthner et al. ${ }^{23}$

\section{Summary}

In this article, we have demonstrated that water-soluble n-PDI and p-PDI compounds can be used to assemble films that can efficiently transfer energy to a trapping layer composed of n-TDI (Scheme 1). The characteristic distance for energy transfer is model-dependent, but approximately the same value (ca. 5.4 $\mathrm{nm}$ ) is extracted from either the Kuhn J-aggregate model (eq 4 with $x=2$ ) or the Kenkre-Wong exciton model (eq 8). Although this characteristic distance is on the order of the Förster $R_{0}$ value for n- or p-PDI energy transfer to n-TDI (Table 1), we discount single-step energy transfer from a trapped exciton (eq 3 or 4 with $x=4$ ) because of the relatively poor fit to the data. We cannot dismiss the Kuhn model applied to an exciton trapped on the outermost layer, but the fit to this model is less satisfactory than if we were to consider the exciton to be homogeneously distributed over the volume of the film.
The characteristic distance of energy transfer is considerably smaller than has been reported by Gregg et al. ${ }^{11}$ or Adams et al. ${ }^{12}$ It remains to be seen if this is related to the preparation or processing method of an MLBL film or if this is an intrinsic property of our particular n- and p-PDI moieties themselves. It seems likely to us that long-range energy (and charge) transport could be achieved with a combination of charged PDI species (perhaps with different chemical structures) and the MLBL approach (perhaps with appropriate annealing steps).

Acknowledgment. S.E.W. thanks the Welch Foundation Program (grant F-356) for its financial support of this work. K.M. acknowledges the support of the Deutsche Forschungsgemeinschaft (Sonderforschungsbereich 625 and Schwerpunktprogramm Organische Feldeffekttransistoren).

Supporting Information Available: Experimental $\Phi_{\mathrm{ET}}$ data and best-fit comparison, weighted differences $\left(\left(\Phi_{\mathrm{ET}}^{\text {expt }}-\Phi_{\mathrm{ET}}^{\text {calc }}\right) / \Phi_{\mathrm{ET}}^{\text {expt }}\right)$, and a plot of weighted differences versus $n$ illustrating the degree of correlation in different fits. This material is available free of charge via the Internet at http://pubs.acs.org.

LA0634903 